\title{
Anomalous Grain Growth in a Polycrystalline Monolayer of Colloidal Hard Spheres
}

\author{
François A. Lavergne, ${ }^{*}$ Dirk G. A. L. Aarts, and Roel P. A. Dullens \\ Department of Chemistry, Physical and Theoretical Chemistry Laboratory, University of Oxford, \\ South Parks Road, Oxford OX1 3QZ, United Kingdom
}

(Received 8 June 2017; revised manuscript received 13 October 2017; published 14 December 2017)

Understanding grain growth is key for controlling the microstructure and the mechanical properties of most polycrystalline materials, including metals, alloys, and ceramics. However, the precise mechanisms and kinetics of grain growth remain poorly understood both at the theoretical level and experimentally as direct observation is cumbersome in atomic systems. Here, we study the grain growth process in a polycrystalline monolayer of colloidal hard spheres. We find that the bond-orientational correlation function satisfies the dynamic scaling hypothesis and has the general scaling form predicted for systems containing random domain walls. However, the associated correlation length grows slower than $\sim t^{1 / 2}$, which corresponds to normal curvature-driven grain growth. To understand the origin of this anomalous grain growth, we directly monitor the evolution of the grain boundary network by measuring the so-called grain boundary character distribution. We show that there is a strong annihilation of large-angle grain boundaries while small-angle grain boundaries become relatively more present. Using scaling arguments, we derive the time dependence of the correlation length and show its good agreement with the data. We conclude that the origin of anomalous grain growth is the curvature-driven coarsening of the large-angle grain boundaries at a rate that depends on their relative length in the total grain boundary network.

DOI: 10.1103/PhysRevX.7.041064

\section{INTRODUCTION}

Coarsening phenomena correspond to the nonequilibrium relaxation of a system following a quench from the disordered phase into the ordered phase by quickly varying an external control parameter such as the temperature $[1,2]$. Ordering is achieved by the annihilation of defects, commonly point defects or domain walls [3,4], separated by a typical distance $R(t)$ called the growth length, which increases as a function of time $[1,2]$. Its time dependence is usually a power law $R(t) \sim t^{\alpha}$, referred to as the growth law, which depends on the mechanism of coarsening [2]. Analytically finding the value of the exponent $\alpha$ from the growth mechanism is usually a hard task because of the complexity of the system [1]. However, in some cases, the growth laws can be derived theoretically and have been verified experimentally. Examples include disclination-driven ordering in striped patterns $(\alpha=1 / 4)$ [5], spinodal decomposition in colloid-polymer mixtures $(\alpha=1)$ [6], and curvature-driven growth in liquid crystals $(\alpha=1 / 2)[7,8]$.

\footnotetext{
*francois.lavergne@lincoln.oxon.org

†roel.dullens@chem.ox.ac.uk
}

Published by the American Physical Society under the terms of the Creative Commons Attribution 4.0 International license. Further distribution of this work must maintain attribution to the author(s) and the published article's title, journal citation, and DOI.
Subject Areas: Condensed Matter Physics,

Materials Science, Soft Matter
Grain growth is a particular kind of coarsening process, which is encountered in polycrystalline materials that are composed of adjacent crystalline domains of mismatching orientations, called grains, and separated by domain walls, termed grain boundaries (GBs) [9]. As the mechanical properties of polycrystalline materials are directly related to the size of the constituent crystalline grains [10-12], understanding the underlying grain growth mechanism is key in controlling them. Grain growth has been studied extensively in simulations [13-17] and in experiments on metallic systems $[18,19]$. Nevertheless, the mechanisms for grain growth are still far from established, and it is in fact possible that multiple mechanisms may contribute concurrently $[16,17,20-23]$. It is often assumed that grain growth is purely driven by the curvature of GBs $[9,24,25]$, which leads to a growth law of $t^{1 / 2}$ and is referred to as "normal" grain growth $[9,24]$. However, the growth law is often found to be different from $t^{1 / 2}$ [9], which is referred to as "anomalous" grain growth [26]. Variability in the growth exponents was observed for various metals $[9,27]$, but also for model systems, such as repulsive colloids [28,29] and block-copolymer systems [30-32]. The possibility of deriving a growth law for the case of grain growth is still an open question because of uncertainties about the growth mechanisms involved.

Colloidal polycrystalline monolayers are very convenient model systems to study grain growth since they can be imaged by means of simple optical microscopy at singleparticle resolution during a significant period of time, 
which is crucial for probing the coarsening dynamics with sufficient accuracy [29,33-35]. Colloidal hard spheres confined to a monolayer form a hexagonal close-packed crystal when their area fraction exceeds the value 0.73 [3638]. Quenching the system into the crystal phase results in a polycrystalline monolayer, with randomly oriented grains $[39,40]$, structurally analogous to polycrystalline graphene sheets [41]. The grains are enclosed by GBs that are characterized by (i) the difference in orientation between the two adjacent crystals, called the "misorientation," and (ii) the orientation of the GB line, called the "inclination" [25]. The GBs form a network whose detailed structure can be specified by the relative amount of GBs of each misorientation and inclination. This is quantified by the "grain boundary character distribution" (GBCD), which is the central link between the microscopic details and the macroscopic properties of the GB network, such as the average misorientation, inclination, or grain size $[42,43]$. Hence, the time evolution of the GBCD is at the heart of the grain growth process.

In this work, we elucidate the origin of anomalous grain growth observed in a polycrystalline monolayer of colloidal hard spheres. The bond-orientational ordering process satisfies dynamic scaling with a good agreement between the experimental bond-orientational correlation function and the general scaling form predicted for systems containing random domain walls. Nevertheless, the bondorientational correlation length is found to grow slower than expected for curvature-driven coarsening, suggesting the presence of anomalous grain growth. By directly measuring the GBCD, we show that the grain growth kinetics strongly depends on the misorientation of GBs but is not affected by their inclination. In particular, we find a preferential annihilation of GBs with a high misorientation, while the relative length of GBs with a low misorientation increases. In addition, we find that the GBs with a small misorientation are found to have little impact on the decay of the bond-orientational correlation function. We derive the scaling law for the correlation length and find good agreement with the experimental data. This suggests that the origin of anomalous grain growth lies in the fact that the rate of coarsening of GBs with a high misorientation depends on their relative length in the total GB network.

\section{THEORY}

\section{A. Normal grain growth}

The migration velocity $v$ of a GB is usually related to the GB mobility $M$ and the driving force $P$ per unit length of a GB (in 2D) via the Herring relation $[25,44]$

$$
v=M P,
$$

where $M$ can be seen as the inverse of a friction coefficient per unit length of a GB. In the case where the curvature of the GB is the only driving force, one has $P=\Gamma \kappa$, with $\kappa$ the local curvature and $\Gamma$ the GB stiffness $[25,44]$. The GB stiffness is an energy per unit length (in 2D) given by $\Gamma=\gamma+\gamma^{\prime \prime}$, where $\gamma$ is the interfacial free energy of the GB and $\gamma^{\prime \prime}$ is its second derivative with respect to the GB inclination [44]. Rewriting the Herring relation for the case of curvature-driven GB migration yields

$$
v=M \Gamma \kappa,
$$

which is equivalent to the Allen-Cahn equation for curvature-driven domain wall migration $[1,24]$. The product $M \Gamma$, called the "reduced mobility" and denoted $M^{*}$ in the following [45], has the dimension of an area per unit time. It corresponds to the migration rate of a $\mathrm{GB}$ as it is the typical area spanned by a moving GB per unit time.

In a polycrystalline material, where GBs form a network, the time dependence of the average grain size, $R(t)$, can be obtained from Eq. (2). Assuming that $v \sim \mathrm{d} R / \mathrm{d} t$ and $\kappa \sim 1 / R$, one obtains

$$
\frac{\mathrm{d} R}{\mathrm{~d} t} \sim \frac{M^{*}}{R} .
$$

This can be integrated to give

$$
R(t) \sim t^{1 / 2},
$$

which is known as the growth law of the Allen-Cahn universality class $[1,2,24]$ or "normal" grain growth [9].

\section{B. Anomalous grain growth}

In most polycrystalline materials, however, normal grain growth is not observed as the grain size increases with an exponent different from $1 / 2$ [9], which is referred to as "anomalous" grain growth [26]. Indeed, as GBs are solidsolid interfaces, their mobility $M$, their stiffness $\Gamma$, and thus their reduced mobility $M^{*}$ depend on both the misorientation $\theta$ between the two crystals and the inclination $\psi$ of the GB $[9,25]$. Though this does not modify the local GB dynamics as described by Eq. (2), it does imply that GBs with different misorientations and inclinations coarsen at different rates, i.e., that the growth law depends on the detailed structure of the GB network. The latter can be described by the so-called GBCD, denoted $p(\theta, \psi, t)$, which measures the length of GBs with a given misorientation $\theta$ and inclination $\psi$ at time $t, l(\theta, \psi, t)$, divided by the total length of the GB network in the system $[42,43]$ :

$$
p(\theta, \psi, t)=\frac{l(\theta, \psi, t)}{\int_{I_{\theta}} \int_{I_{\psi}} l(\theta, \psi, t) \mathrm{d} \psi \mathrm{d} \theta} .
$$

Here, $I_{\theta}\left(I_{\psi}\right)$ denotes the interval of the possible values of $\theta(\psi)$.

The GBCD is the fundamental quantity that relates the details of the microstructure to average quantities 
(a)

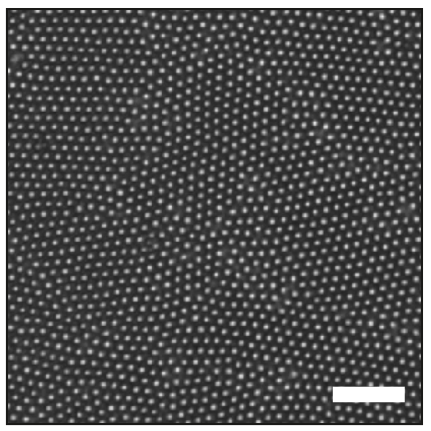

(d)

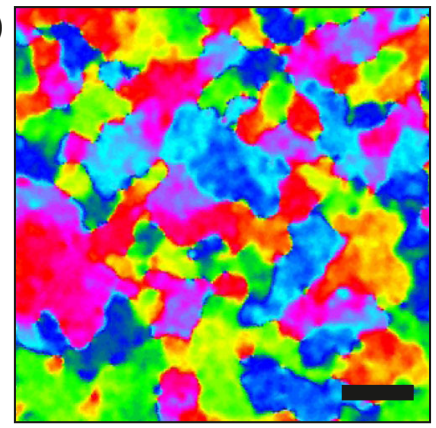

(b)

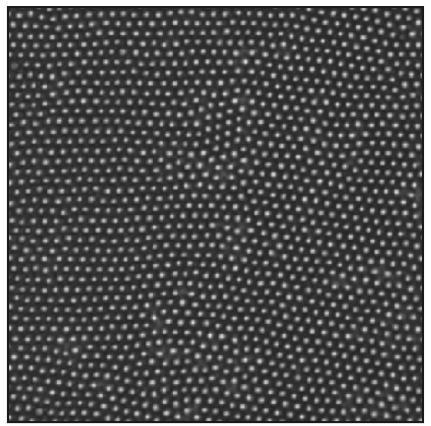

(e)

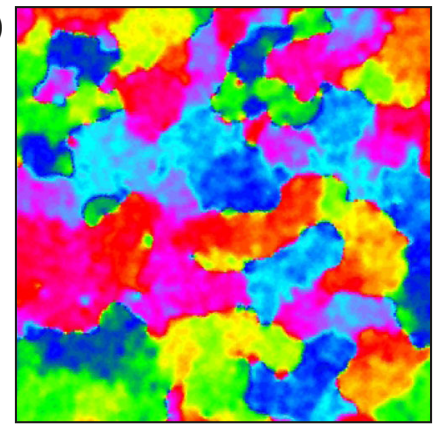

(c)

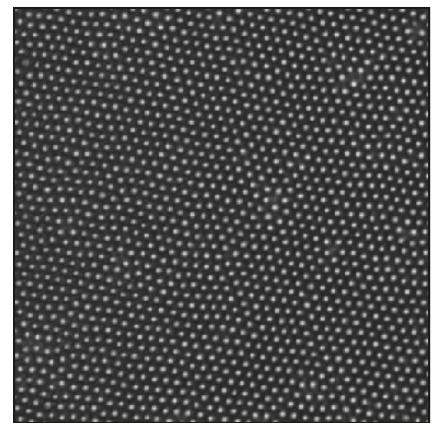

(f)

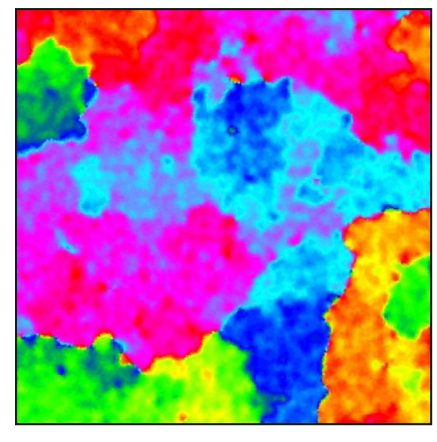

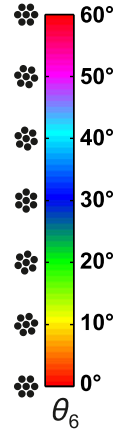

FIG. 1. (a)-(c) Microscopy images of the colloidal polycrystalline monolayer at different times during grain growth: (a) $32 t_{B}$, (b) $100 t_{B}$, and (c) $1000 t_{B}$. The scale bar represents $20 \mu \mathrm{m}$. (d)-(f) The corresponding smoothed orientation fields (full field of view) at the same times. The scale bar represents $100 \mu \mathrm{m}$.

describing the GB network $[42,43,46]$. For example, from the misorientation and inclination-dependent reduced mobility of each boundary $M^{*}(\theta, \psi)$, one can find its time-dependent average in the system as

$$
\left\langle M^{*}\right\rangle(t)=\int_{I_{\theta}} \int_{I_{\psi}} M^{*}(\theta, \psi) p(\theta, \psi, t) \mathrm{d} \psi \mathrm{d} \theta,
$$

which thus depends on the detailed structure of the GB network through $p(\theta, \psi, t)$.

For the case that the grain size $R(t)$ is the only relevant length scale in the growth process, one can expect-analogous to normal grain growth described by Eq. (3) - that at late times a scaling regime is reached where the grain size evolves according to [46]

$$
\frac{\mathrm{d} R}{\mathrm{~d} t} \sim \frac{\left\langle M^{*}\right\rangle}{R} .
$$

As $\left\langle M^{*}\right\rangle$ is a function of time, one expects deviations from the law of normal grain growth, i.e., $R(t)$ does not scale like $t^{1 / 2}$ anymore. Note that if the GBCD does not depend on time, $\left\langle M^{*}\right\rangle$ is constant and integration of Eq. (7) leads back to the law of normal grain growth, Eq. (4).

\section{RESULTS AND DISCUSSION}

\section{A. Bond-orientational ordering}

A two-dimensional colloidal hard sphere system $[38,47]$ is quenched into the crystalline phase [36], which results in the formation of a polycrystalline monolayer subsequently monitored using video microscopy (see Appendix A) [39,40]. The quench is achieved by the rapid sedimentation-inherent to the large density mismatch between the particles and the solvent-of the colloidal particles to the bottom surface of the sample cell, causing the in-plane area fraction to increase from 0 to 0.73 within a few minutes only. Figures 1(a)-(c) show representative microscopy images of a small area of the colloidal polycrystalline monolayer at different times, indicating local hexagonal bond-orientational order and an evident increase of the average grain size. The experimental time $t$ is defined such that $t=0$ corresponds to the injection of the particles in the sample cell, i.e., the time of the start of the quench, and we analyze the data from the moment the monolayer has formed, typically a few minutes after injection. Note that time is expressed in units of the Brownian time, the time a colloidal sphere takes to diffuse over its own diameter at infinite dilution, $t_{B}=28 \mathrm{~s}$ in our case (see Appendix A). The polycrystalline structure is clearly revealed in Figs. 1(d)-1(f) by the color maps of the smoothed local orientation field $[39,40]$ (see Appendix B) for the whole field of view, where the color of a grain corresponds to the local orientation of the crystallite. Consistent with the microscopy images in Figs. 1(a)-1(c), the average grain size clearly increases, and the total grain boundary length decreases over time, indicating that significant grain growth takes place within the time scale of our experiments. 
(a)

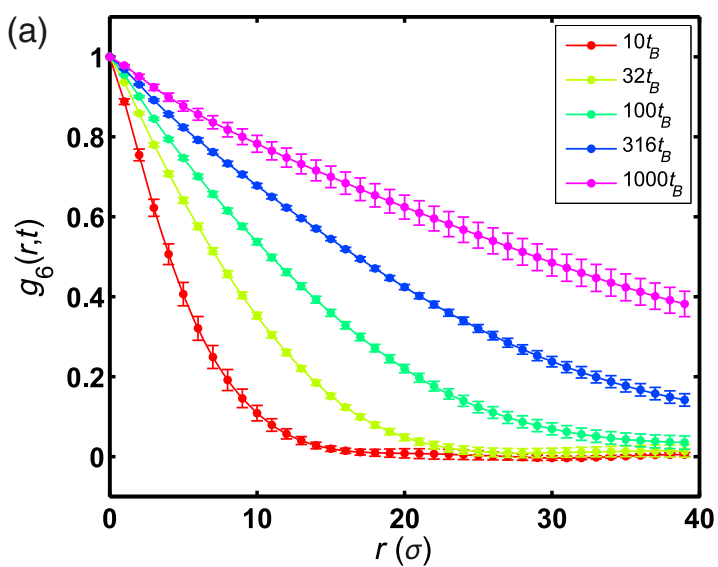

(c)

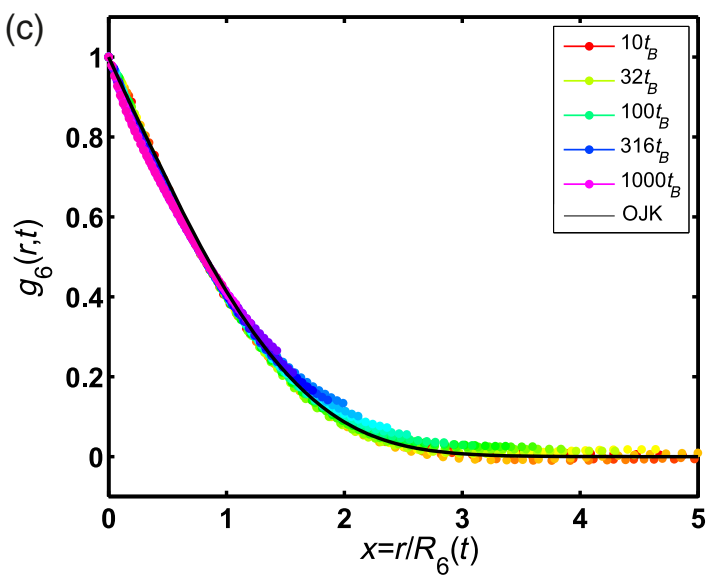

(b)

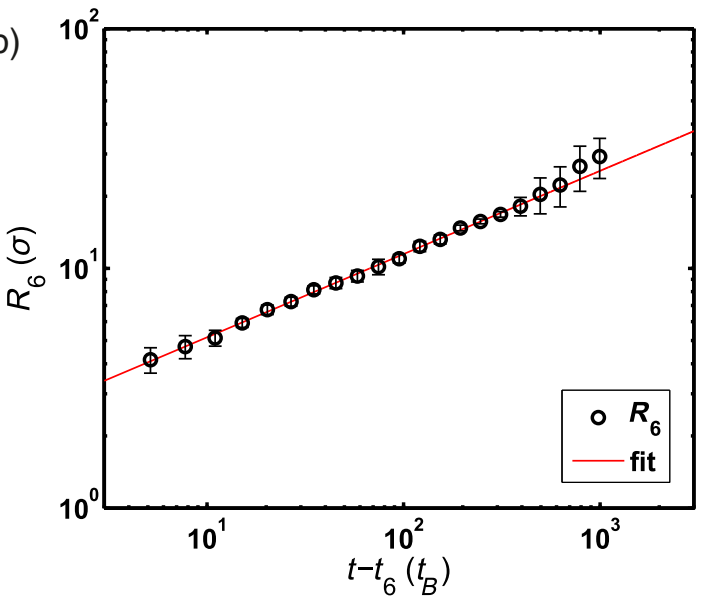

(d)

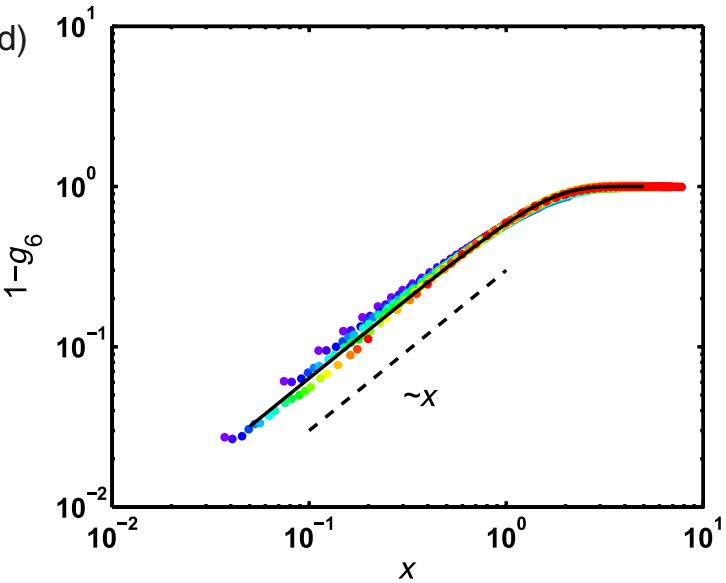

FIG. 2. (a) The bond-orientational correlation function $g_{6}(r, t)$ as defined by Eq. (B3) for different times. (b) Time-evolution of the bond-orientational correlation length $R_{6}$. The red solid line is the result of the fit to $R_{6} \sim\left(t-t_{6}\right)^{\alpha_{6}}$. All error bars correspond to the standard deviation of the different experimental runs. (c) The correlation function $g_{6}$ as a function of the distance rescaled by the correlation length $R_{6}$. The solid black line is the correlation function given by OJK theory; see Eq. (8). (d) A log-log plot of $1-g_{6}$ as a function of the rescaled distance $x=r / R_{6}(t)$, which scales as $x$ (black dashed line) following the prediction from Porod's law; see Eq. (9).

The grain growth process is quantitatively monitored using the bond-orientational correlation function, $g_{6}(r, t)$, computed using the normalized smoothed bond-orientational order parameter $\hat{\psi}_{6}$ to accurately probe the correlations in the presence of GBs (see Appendix B). The behavior of $g_{6}$ is shown in Fig. 2(a) as a function of the distance, $r$, and for different values of the time, $t$. The range of the bondorientational correlations clearly increases with time, indicating the growing extent of the bond-orientational order in the system, consistent with the images and orientation fields in Figs. 1(a)-1(c) and 1(d)-1(f). A bond-orientational correlation length $R_{6}$ is defined by $g_{6}\left(R_{6}, t\right)=0.5$ and is determined at all times $[8,48]$. The time dependence of $R_{6}$ is shown in Fig. 2(b) and is fitted by $R_{6} \sim\left(t-t_{6}\right)^{\alpha_{6}}$ following Ref. [7], where $t_{6}$ is the time at which the system enters the scaling regime. The fit gives $t_{6}=5 t_{B}$, which roughly corresponds to the time required for the monolayer to form. This indicates that the scaling regime is entered almost immediately after the monolayer has formed and, as such, that $t_{6}$ is very close to-but not defined as - the moment that coarsening starts. Because of its relatively small value compared to the time scale of coarsening, the time $t_{6}$ quickly becomes irrelevant and the growth can be considered solely in terms of the time $t$. Importantly, the growth exponent is found to be $\alpha_{6}=0.35 \pm 0.02$, which is lower than the exponent expected for normal grain growth, $\alpha=1 / 2$, as in Eq. (4) $[1,8,48]$.

Plotting $g_{6}$ as a function of the rescaled distance $r / R_{6}$ shows an excellent collapse of the data [Fig. 2(c)], indicating that the dynamic scaling hypothesis $[1,2]$ is satisfied by $g_{6}$, i.e., $g_{6}(r, t)=f\left(r / R_{6}(t)\right)$, where $f$ is a time-independent scaling function. This indicates that the domain structure is self-similar as long as the distances are rescaled by the growth length $R_{6}$, which is the only relevant length scale in the bond-orientational ordering process $[1,2]$. Note that redefining $R_{6}$ by changing the constant in the right-hand side of $g_{6}\left(R_{6}, t\right)=0.5$ does not affect these results as the new growth length is then proportional to the former one as a consequence of dynamic scaling. 
(a)

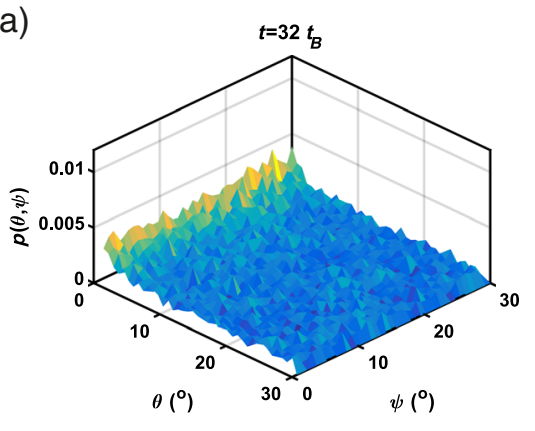

(b)

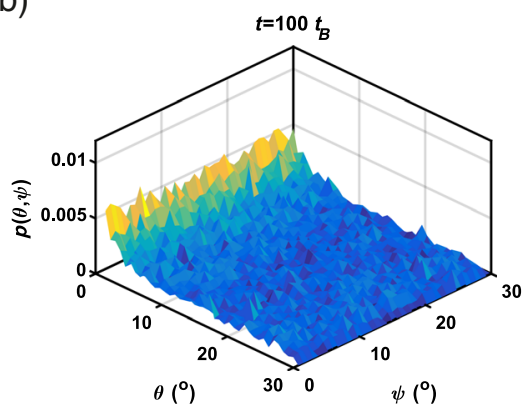

(c)

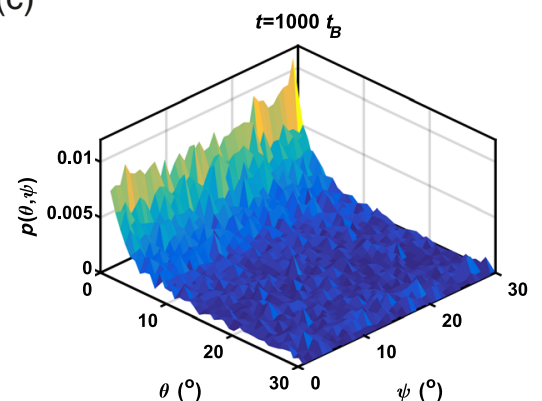

(d)

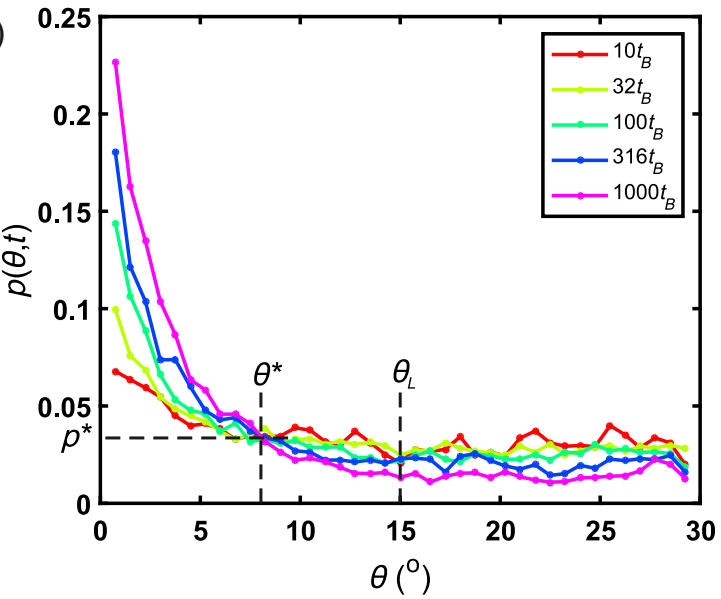

(e)

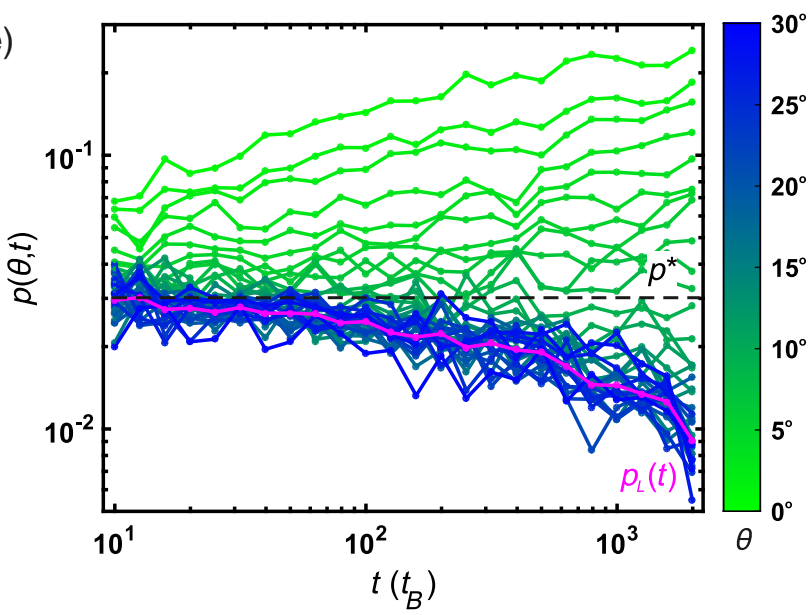

FIG. 3. (a)-(c) Grain boundary character distribution (GBCD), $p(\theta, \psi, t)$, as a function of the misorientation, $\theta$, and the inclination, $\psi$, for three different times during coarsening. (d) The $\psi$-averaged GBCD, $p(\theta, t)$, as a function of the misorientation, $\theta$, for different times during coarsening. The LAGB regime is defined as GBs with misorientations above $\theta_{L}$. The value $\theta^{*}$ is a crossover point in the SAGB regime corresponding to the value $p^{*}$ of the GBCD. (e) GBCD as a function of time for different misorientations, where each curve is colored according to the misorientation as indicated by the color bar. The time dependence of the GBCD is strongly misorientation dependent below $\theta_{L}$, involving a transition from an increasing to a decreasing GBCD at the crossover value $\theta^{*}$, where the GBCD equals $p^{*}$. Above $\theta_{L}$, the GBCD curves decay in the same fashion, which corresponds to an average master curve denoted $p_{L}(t)$ (magenta line).

We compare the scaling form of $g_{6}$ to Ohta-JasnowKawasaki (OJK) theory, which states that the correlation function for a growth process driven by the motion of random domain walls after a quench into the ordered phase is given by $[49,50]$

$$
C_{\mathrm{OJK}}(x)=\frac{2}{\pi} \arcsin \left(e^{-x^{2} / 2}\right) .
$$

Here, $x=r / R(t)$ is the distance in units of a timedependent growth length $R(t)$. As $R_{6}$ is the only relevant length scale, Eq. (8) is directly tested by plotting $g_{6}$ as a function of $x=r / R_{6}(t)$ in Fig. 2(c). The excellent agreement between $g_{6}\left(r / R_{6}\right)$ and $C_{\mathrm{OJK}}$ in Fig. 2(c) at all values of $x$ suggests that the GBs act as domain walls in the orientation field. At short distances or late times, which is equivalent to small values of $x$, the correlation function behaves as $C_{\mathrm{OJK}}(x) \simeq 1-2 x / \pi[50,51]$. This leads to a linear decay of the correlations

$$
g_{6}(r, t) \simeq 1-\frac{2}{\pi} \frac{r}{R_{6}(t)},
$$

which is the real-space counterpart of the well-known Porod's law $[1,50,51]$. We directly test this feature by plotting $1-g_{6}$ in terms of $x=r / R_{6}(t)$, as shown in Fig. 2(d). The data exhibit a very clear linear behavior as $x \rightarrow 0$, which is in excellent agreement with Porod's law. As expected, Porod's law breaks down at larger $x$, where the full OJK expression, Eq. (8), needs to be considered. Note that both the OJK scaling form and Porod's law were found to be valid in coarsening systems containing random domain walls such as Potts models [48] and liquid crystals [7,8]. Finally, we stress that the OJK form in Eq. (8) is noticeably different from the expressions of $g_{6}$ in each of the equilibrium phases of hard disks in 2D space, namely, liquid, hexatic, and crystal, for which $g_{6}$ is, respectively, a decaying exponential, a power law, and a constant $[4,36-38,52,53]$.

\section{B. Grain boundary character distribution}

The fact that the growth exponent, $\alpha_{6} \simeq 0.35$, is different from $1 / 2$ suggests the presence of anomalous grain growth in this system [26], which points towards a dependence of the reduced mobility on the misorientation and inclination, $M^{*}(\theta, \psi)$, as can be seen from Eqs. (6) and (7). 
(a)

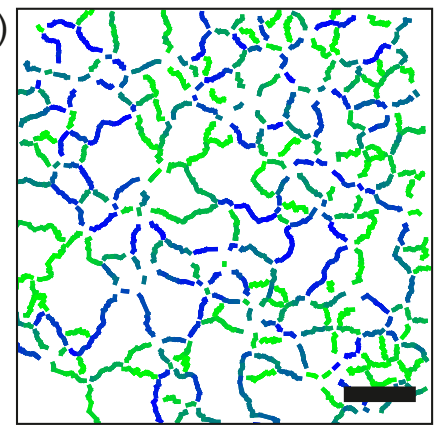

(b)

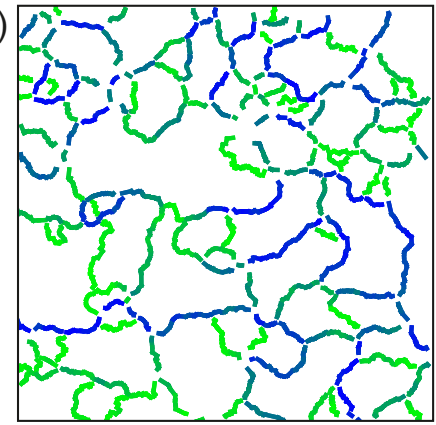

(c)

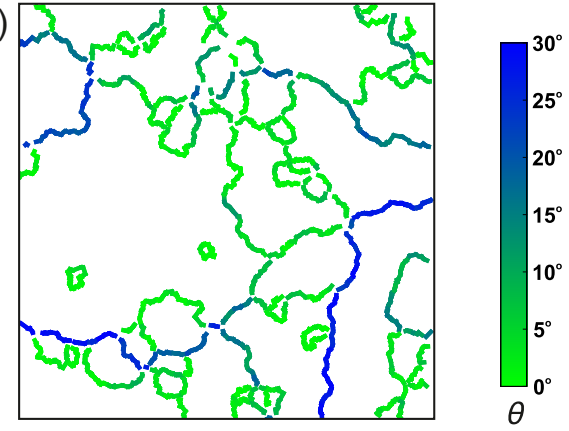

FIG. 4. (a)-(c) View of the GBs colored according to the value of their misorientation at the exact same times as in Figs. 1(a)-1(c) and 1(d)-1(f). This provides a direct way to visualize the SAGBs and LAGBs during coarsening. The scale bar represents $100 \mu \mathrm{m}$.

In order to check whether the kinetics of GBs depend on their misorientation, we monitor the relative length of GBs with a given misorientation $\theta$ and inclination $\psi$ by directly computing the GBCD, $p(\theta, \psi, t)$ (see Appendix C). To obtain $p(\theta, \psi, t)$, we sum the lengths of GB portions with a misorientation in an interval $[\theta ; \theta+\delta \theta]$ and an inclination in an interval $[\psi ; \psi+\delta \psi]$ (with $\delta \theta$ and $\delta \psi$ small), and divide by the total length of the GB network at time $t$ (see Appendix C). Surface plots of the GBCD are shown in Figs. 3(a)-3(c) for three different times during coarsening. There is a clear increase of the GBCD at low misorientations with time, but strikingly, the GBCD does not change as a function of the inclination at all times. This may originate from the fact that GBs are curved and exhibit thermal fluctuations [54], allowing the inclinations to be equiprobable. As the inclination $\psi$ is not affecting the coarsening kinetics, the GBCD can in fact be treated as a function of the misorientation $\theta$ only. In the following, we consider the $\psi$-averaged GBCD, $p(\theta, t)=\int_{I_{\psi}} p(\theta, \psi, t) \mathrm{d} \psi$, and merely refer to it as "the GBCD" for simplicity. The same applies to the $\psi$-averaged reduced mobility, $M^{*}(\theta)=$ $\int_{I_{\psi}} M^{*}(\theta, \psi) \mathrm{d} \psi$.

The GBCD as a function of the misorientation $\theta$ only is shown in Fig. 3(d), for different times during coarsening. Additionally, the GBCD is also plotted as a function of time for different values of the misorientation in Fig. 3(e). Clearly from Fig. 3(d), the GBCD is a decaying function of $\theta$ with a plateau at high $\theta$, typically above a value $\theta_{L} \simeq 15^{\circ}$. The height of the plateau decreases as a function of time, which is clear from the time decay of the GBCD curves for $\theta \geq \theta_{L} \simeq 15^{\circ}$ in Fig. 3(e). Strikingly, one can see from Fig. 3(e) that for misorientations above $\theta_{L} \simeq 15^{\circ}$, the GBCD becomes independent of the misorientation, so that

$$
p(\theta, t)=p_{L}(t) \quad \text { for } \theta \geq \theta_{L} .
$$

Experimentally, we determine $p_{L}(t)$ by averaging the GBCD over the interval $\left[\theta_{L}, 30^{\circ}\right]$, as shown by the purple line in Fig. 3(e). One concludes that GBs with a misorientation greater than $\theta_{L} \simeq 15^{\circ}$ have the same kinetics in the GB network, as it is governed by $p_{L}(t)$, which does not depend on $\theta$. In the following, GBs with a misorientation $\theta \in\left[\theta_{L} ; 30^{\circ}\right]$, where $\theta_{L}=15^{\circ} \pm 2^{\circ}$, are referred to as "large-angle grain boundaries" (LAGBs). Otherwise they are termed "small-angle grain boundaries" (SAGBs).

Interestingly, the kinetics of SAGBs strongly contrasts with that of LAGBs. Indeed, the combined inspection of Figs. 3(d) and 3(e) reveals that for misorientations below $\theta_{L}$, the GBCD can increase or decrease depending on whether $\theta$ is smaller or larger than a crossover value $\theta^{*}$. From the data in Fig. 3(d), we estimate the location of the crossover to be $\theta^{*}=8.5^{\circ} \pm 1^{\circ}$, which corresponds to a constant value of the GBCD, $p^{*} \simeq 0.03$, as can be seen from Fig. 3(e). This shows that unlike LAGBs, the kinetics of SAGBs in the GB network strongly depends on the value of their misorientation.

\section{Kinetics of the grain boundary network}

To clarify the contributions of LAGBs and SAGBs to the coarsening kinetics, the GB network is directly visualized in Figs. 4(a)-4(c), which shows snapshots of the GBs colored according to the magnitude of their local misorientation $\theta$ for the whole field of view (see Appendix C). These snapshots indicate a significant annihilation of LAGBs, leaving large areas containing mainly SAGBs, which enclose smaller grains. This can be quantified by monitoring the evolution of the total lengths of GBs, SAGBs, and LAGBs, defined by

$$
\Lambda_{k}=\int_{k} l(\theta, t) \mathrm{d} \theta,
$$

with $k$ corresponding to $I=\left[0 ; 30^{\circ}\right], S=\left[0 ; \theta_{L}\right)$, and $L=\left[\theta_{L} ; 30^{\circ}\right]$, respectively, and such that $\Lambda_{I}=\Lambda_{S}+\Lambda_{L}$. The time evolution of the $\Lambda_{k}$ 's is shown in Fig. 5(a). While $\Lambda_{I}$ decreases slightly over time, there is a strong decrease of $\Lambda_{L}$, indicating significant annihilation of LAGBs, consistent with the behavior of the GBCD in Figs. 3(d) and 3(e). This is in clear contrast with the behavior of $\Lambda_{S}$, which increases very weakly throughout the process. From this, we deduce that the SAGBs become relatively more present as they constitute the majority of the grain boundaries at long times. 

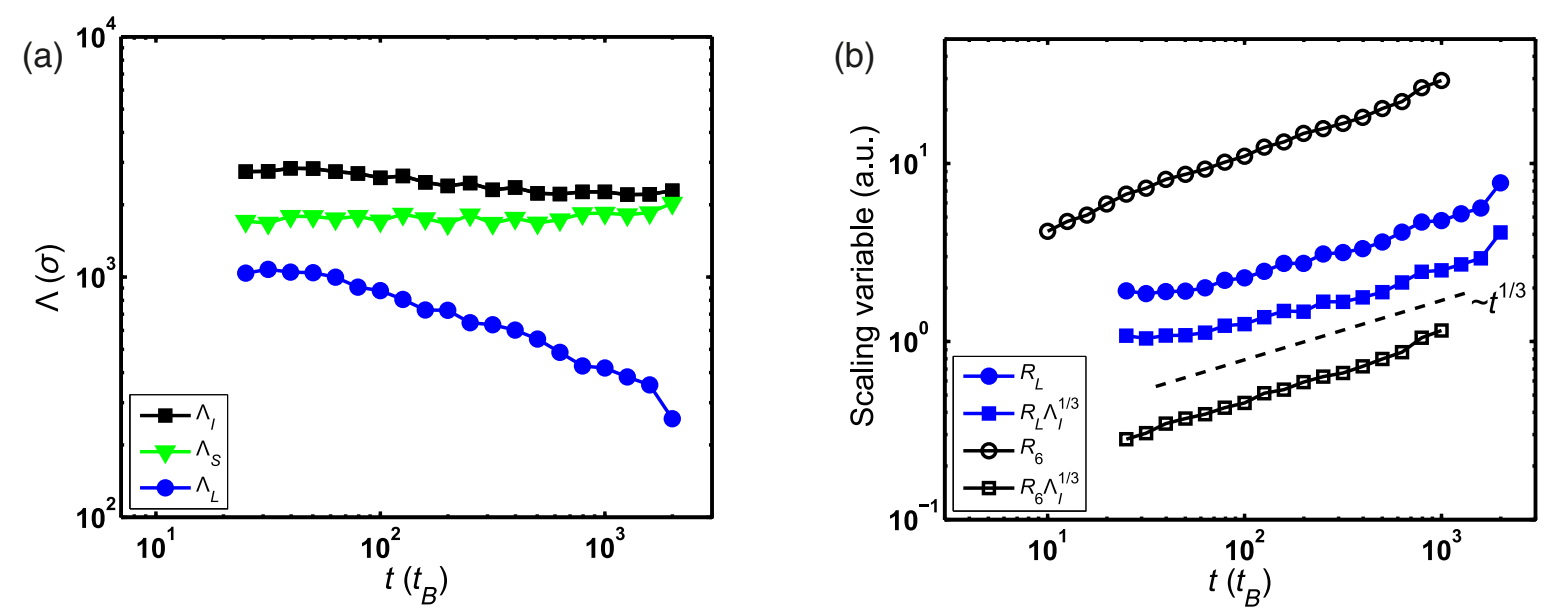

FIG. 5. (a) Time evolution of the total lengths of GBs, SAGBs, and LAGBs, namely, $\Lambda_{I}, \Lambda_{S}$, and $\Lambda_{L}$, in units of particle diameters. (b) Time evolution of the length scale $R_{L}$, obtained from $A / \Lambda_{L}$, where $A$ is the area of the field of view. The quantity $R_{L} \Lambda_{I}^{1 / 3}$ behaves as $t^{1 / 3}$ (black dashed line) at long times. The bond-orientational correlation length, $R_{6}$, exhibits the same scaling properties as $R_{L}$. The offsets of the curves are arbitrary to facilitate visualization and the differences in time range are attributable to difficulties in accessing $R_{6}$ $\left(R_{L}\right.$ and $\left.\Lambda_{I}\right)$ accurately at long (short) times.

In light of the snapshots in Figs. 4(a)-(c) and the differences in the kinetics between SAGBs and LAGBs visible in Fig. 5(a), the network of GBs can be seen as having two components: the LAGBs, which are in the minority, and the SAGBs. Based on this, we define two typical length scales,

$$
R_{k}=\frac{A}{\Lambda_{k}}
$$

where $A$ is the area of the field of view and $k=S, L$. Within this definition, $R_{S}\left(R_{L}\right)$ corresponds to the average distance from one SAGB (LAGB) to another. It is important to note that $R_{S}$ and $R_{L}$ are different from the grain size, $R_{I}=A / \Lambda_{I}$, which is the mean distance from one GB to another, regardless of their types.

In order to derive the growth laws corresponding to $R_{S}$ and $R_{L}$, it is reasonable to assume that the kinetics of SAGBs and LAGBs are driven by the curvature of the GBs of their own type. Consequently, the kinetics can be described by two equations analogous to Eq. (7) corresponding to each network as

$$
\frac{\mathrm{d} R_{k}}{\mathrm{~d} t}=\frac{\left\langle M^{*}\right\rangle_{k}}{R_{k}} .
$$

Here, the $\left\langle M^{*}\right\rangle_{k}$ 's with $k=S, L$ are two time-dependent reduced mobilities defined by

$$
\left\langle M^{*}\right\rangle_{k}(t)=\int_{k} M^{*}(\theta) p(\theta, t) \mathrm{d} \theta
$$

and such that

$$
\left\langle M^{*}\right\rangle_{I}=\left\langle M^{*}\right\rangle_{S}+\left\langle M^{*}\right\rangle_{L},
$$

where $\left\langle M^{*}\right\rangle_{I}$ is the total reduced mobility. Note that all $\left\langle M^{*}\right\rangle_{k}$ 's with $k=I, S, L$ represent time-dependent average coarsening rates, which depend on the configuration of the GB network via the GBCD, $p(\theta, t)$. As they are averaged over many GBs belonging to either of the different groups $I, S$, and $L$, they differ from the misorientation-dependent reduced mobilities $M^{*}(\theta)$ of single GBs.

From the experimental data in Fig. 5(a), it is clear that $\Lambda_{S}$ hardly changes at all, so $\mathrm{d} R_{S} / \mathrm{d} t \simeq 0$, and therefore we focus on the case of LAGBs in Eq. (13). Using successively the definition of $\left\langle M^{*}\right\rangle_{L}$ in Eq. (14) and the property of the GBCD for LAGBs as given by Eq. (10), we obtain

$$
\begin{aligned}
\left\langle M^{*}\right\rangle_{L} & =\int_{L} M^{*}(\theta) p(\theta, t) \mathrm{d} \theta \\
& =p_{L}(t) \int_{L} M^{*}(\theta) \mathrm{d} \theta \\
& \sim p_{L}(t),
\end{aligned}
$$

as the remaining integral over $\theta$ is time independent. Note that, importantly, the last equation means that the behavior of the GBCD for LAGBs, $p_{L}$, is directly proportional to the average reduced mobility of LAGBs, $\left\langle M^{*}\right\rangle_{L}$. As $p_{L}$ decreases during coarsening [see Fig. 3(e)], we deduce that $\left\langle M^{*}\right\rangle_{L}$ decreases as well, without a priori knowledge of $M^{*}(\theta)$.

Finally, we derive the time dependence of $R_{L}$ by combining its definition, $R_{L} \sim 1 / \Lambda_{L}$, with Eq. (11), which gives

$$
R_{L} \sim\left(\int_{L} l(\theta, t) \mathrm{d} \theta\right)^{-1} .
$$


This can be rewritten using successively Eqs. (5), (10), and (11) as

$$
\begin{aligned}
R_{L} & \sim\left(\int_{L} p(\theta, t) \int_{I} l\left(\theta^{\prime}, t\right) \mathrm{d} \theta^{\prime} \mathrm{d} \theta\right)^{-1} \\
& \sim\left(\int_{L} p_{L}(t) \Lambda_{I}(t) \mathrm{d} \theta\right)^{-1} \\
& \sim\left(p_{L}(t) \Lambda_{I}(t)\right)^{-1}
\end{aligned}
$$

since the integrand in the second line does not depend on $\theta$. Combining Eqs. (16) and (18) yields $\left\langle M^{*}\right\rangle_{L} \sim 1 /\left(R_{L} \Lambda_{I}\right)$, which can be inserted into Eq. (13) to give

$$
\frac{\mathrm{d} R_{L}}{\mathrm{~d} t} \sim \frac{1}{\Lambda_{I} R_{L}^{2}} .
$$

Assuming that $R_{L}$ can be described by a power law in the scaling regime probed here, one has $\mathrm{d} R_{L} / \mathrm{d} t \sim R_{L} / t$, and a simple rearrangement of Eq. (19) finally leads to

$$
R_{L} \Lambda_{I}^{1 / 3} \sim t^{1 / 3} .
$$

We directly test the growth law corresponding to Eq. (20) on the experimental data in Fig. 5(b). One can see that the quantity $R_{L} \Lambda_{I}^{1 / 3}$ is parallel to the $\sim t^{1 / 3}$ curve at long times, indicating that Eq. (20) is satisfied. This means that Eq. (13) is valid to describe the kinetics of the LAGB network, and combining this with Eq. (16), $\left\langle M^{*}\right\rangle_{L} \sim p_{L}(t)$, leads to

$$
\frac{\mathrm{d} R_{L}}{\mathrm{~d} t} \sim \frac{p_{L}(t)}{R_{L}} .
$$

Importantly, this implies that the coarsening of the LAGBs is driven by their curvature only, $1 / R_{L}$, but with a timedependent reduced mobility, $p_{L}(t)$, that is nothing but the fraction of LAGBs in the whole GB network at a given time.

As observed in Fig. 5(b), the quantity $R_{6} \Lambda_{I}^{1 / 3}$ obeys the same scaling as $R_{L} \Lambda_{I}^{1 / 3}$, which means that $R_{L}$ and $R_{6}$ correspond to the same length scale [see Fig. 5(b)]. This indicates that the bond-orientational ordering process is equivalent to the coarsening of the LAGB network, suggesting that the bond-orientation correlation function $g_{6}$ is quite insensitive to the presence of SAGBs. Note that $R_{L}$ and $R_{6}$ follow power laws close to $\sim t^{1 / 3}$ [see Fig. 5(b)], indicating that these quantities dominate $\Lambda_{I}$, which decreases only very weakly with time. In fact, the small decrease of $\Lambda_{I}$ implies that $R_{L}$ and $R_{6}$ grow only slightly faster than $t^{1 / 3}$, consistent with the experimental data.

\section{CONCLUSION}

In summary, we have shown that grain growth in a colloidal polycrystalline monolayer of hard spheres is characterized by a strong bond-orientational ordering, which has general scaling features of systems with random domain walls. However, it exhibits anomalous grain growth as the correlation length grows slower than $\sim t^{1 / 2}$. By monitoring the kinetics of the grain boundaries as a function of their misorientation, we have shown the preferential annihilation of large-angle grain boundaries, while the relative length of small-angle grain boundaries increases. The experimental growth law is consistent with curvature-driven coarsening of the large-angle grain boundaries, but with a time-dependent reduced mobility, which depends on their relative length with respect to the whole network of grain boundaries. Finally, the bondorientational ordering process was found to be equivalent to the coarsening of the large-angle grain boundary network, which dominates the decay of the correlations.

The relevance of our results is underlined by the recent surge of interest in two-dimensional materials such as graphene and other atomically thin materials, like silica glasses [55], semiconductor molybdenum disulphide [56,57], or the insulator boron nitrate [58]. The portability of our findings to 3D materials is complex, as the description of GBs in 3D requires a much larger fivedimensional parameter space [25]. However, the scaling of normal grain growth is always $t^{1 / 2}$ regardless of the dimension. While the deviations from normal grain growth that we observe can depend on dimensionality, their physical origin may be the same, thus illustrating the potential use of our approach in 3D materials. We hope that our work will provide a starting point for the development of a theoretical description of the time evolution of the GBCD, based on GB interfacial properties such as their stiffness, mobility, dislocation density, or roughness, for example. This would bridge the gap between the microscopic level and the much larger length scales involved in coarsening that are responsible for the deviations from normal grain growth.

\section{ACKNOWLEDGMENTS}

The European Research Council (ERC) is acknowledged for financial support (ERC Starting Grant No. 279541IMCOLMAT).

\section{APPENDIX A: EXPERIMENTAL METHODS}

Our colloidal model system consists of a dispersion of 2.78- $\mu \mathrm{m}$-diameter melamine-formaldehyde spheres (Microparticles $\mathrm{GmbH}$ ) in a 20/80 v/v\% isopropanol/ water mixture. The stabilization of the dispersion is ensured by the presence of carboxylic-acid surface groups, which give the particles a negative surface charge. The particles are contained in a $200-\mu \mathrm{m}$-thick quartz cell (Hellma Analytics) and because of their high mass density $\left(1.51 \mathrm{~g} / \mathrm{cm}^{3}\right)$, they sediment to the bottom wall of the cell to form a monolayer. The out-of-plane fluctuations are 

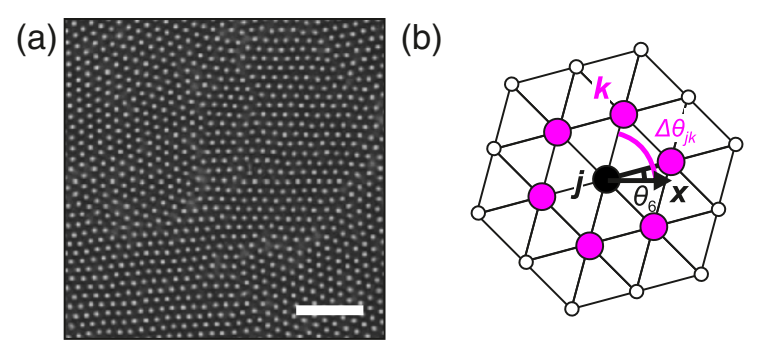

(c)
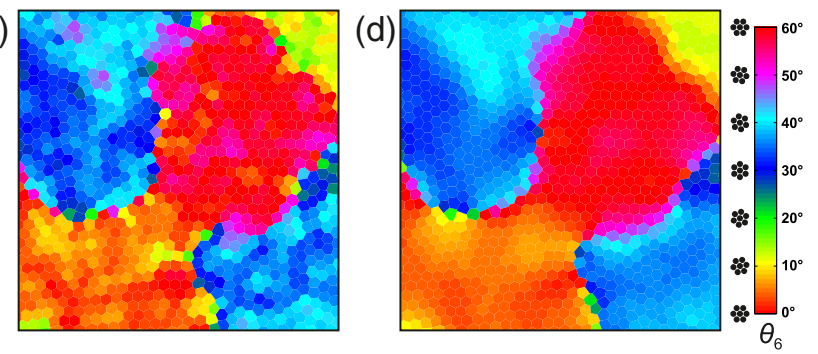

FIG. 6. (a) Microscopy image of a small area of the colloidal polycrystalline monolayer. The scale bar represents $20 \mu \mathrm{m}$. (b) A particle $j$ (black disk) with $k$, one of its six nearest neighbors in the hexagonal lattice (purple disks). The angle between the bond $j-k$ and the $x$ direction is denoted $\Delta \theta_{j k}$, and it is used for the computation of $\psi_{6}$ and the local orientation $\theta_{6}$ (see text). The smoothing of the $\psi_{6}$ field requires the particles in the second shell of nearest neighbors (white disks). (c) Voronoi tessellation, where each cell is colored according to the value of $\theta_{6}$ as indicated by the color bar. (d) Smoothed orientation field $\tilde{\theta}_{6}$.

negligible, resulting in a quasi-two-dimensional system $[38,47]$. In these conditions, our colloidal particles interact as hard spheres, as was recently shown in Refs. [38,47,59]. The system is fully characterized by the area fraction in the midplane of the colloidal particles in the monolayer, $\phi=\pi N \sigma^{2} /(4 A)$, where $\sigma$ is the particle diameter and $N$ is the number of particles located in an area $A$. Because of the high mass density of the particles $\left(1.51 \mathrm{~g} / \mathrm{cm}^{3}\right)$, the formation of a monolayer with a final area fraction $\phi=0.73$ happens within a few minutes only, which thus quenches the system into the crystalline phase [36-38], and the particles assemble in a polycrystalline monolayer with local hexagonal order [39,40]. The subsequent grain growth is monitored using an inverted optical microscope (Olympus CKX41) and a CMOS camera (Ximea XiQ) with a $20 \times$ objective. Images containing $\sim 4 \times 10^{4}$ particles [see Fig. 6(a) for a partial view] are recorded at a frame rate of $1 / \mathrm{min}$ for $16 \mathrm{~h}$ from the time of filling the cell, defined as $t=0$, and five equivalent runs are performed to improve statistics. The particle coordinates are extracted in each frame using standard routines [60]. This procedure is facilitated by adjusting the focus so that a bright dot appears in the middle of each particle, as can be seen in Fig. 6(a). Time is expressed in units of the Brownian time, $t_{B}=\sigma^{2} /\left(4 D_{0}\right)=28 \mathrm{~s}$, where $D_{0}$ is the experimentally determined diffusion coefficient at infinite dilution.

\section{APPENDIX B: BOND-ORIENTATIONAL ORDER ANALYSIS}

The local bond-orientational order parameter $\psi_{6}\left(\vec{r}_{j}, t\right)$ is used to quantify the local crystalline order of a particle $j$ located at $\vec{r}_{j}$ and is defined as $[4,39,40]$

$$
\psi_{6}\left(\vec{r}_{j}, t\right)=\frac{1}{N_{j}(t)} \sum_{k=1}^{N_{j}(t)} e^{i 6 \Delta \theta_{j k}(t)},
$$

where $\Delta \theta_{j k}$ is the angle between the $x$ direction and the bond vector connecting the particle $j$ to one of its $N_{j}$ nearest neighbors $k$, defined using a Delaunay triangulation [see Fig. 6(b)]. The local orientation of the particle $j$ is then given by

$$
\theta_{6}\left(\vec{r}_{j}, t\right)=\frac{1}{6} \arg \left(\psi_{6}\left(\vec{r}_{j}, t\right)\right)
$$

and it varies from $0^{\circ}$ to $60^{\circ}$ as a result of the hexagonal symmetry of the 2D hexagonal close-packed crystal; see Fig. 6(b). A plot of the $\theta_{6}$ field for a local view of the sample is shown in Fig. 6(c) as an example.

The decay of the bond-orientational order as a function of the distance is measured in terms of the bondorientational correlation function, $g_{6}(r, t)$, which requires a specific computation procedure in the case of a polycrystalline sample because of the presence of grain boundaries. First, the field $\psi_{6}$ is smoothed by replacing $\psi_{6}\left(\vec{r}_{i}, t\right)$ with its average over the particles constituting the first two coordination shells of the particle $i$ [see Fig. 6(b)]. The smoothed field is denoted $\tilde{\psi}_{6}\left(\vec{r}_{i}, t\right)$, and the smoothed local orientation field is defined by $\tilde{\theta}_{6}=\arg \left(\tilde{\psi}_{6}\right) / 6$; see Fig. 6(d) for an illustration. This operation prevents the correlation function from sharply dropping at small distances because of noise in the $\psi_{6}$ field [8]. The field $\tilde{\psi}_{6}$ is then normalized according to $\hat{\psi}_{6}=\tilde{\psi}_{6} /\left|\tilde{\psi}_{6}\right|$, which enables a more accurate probing of the correlations at short distances [51]. Eventually, the bond-orientational correlation function $g_{6}(r, t)$ is computed as

$$
g_{6}(r, t)=\operatorname{Re}\left(\left\langle\hat{\psi}_{6}^{*}\left(\vec{r}+\vec{r}_{0}, t\right) \hat{\psi}_{6}\left(\vec{r}_{0}, t\right)\right\rangle\right),
$$

where $\langle$.$\rangle denotes the average over all the pairs of particles$ separated by a distance $r$. Within this definition, $g_{6}(r, t)$ is simply the average cosine of $6\left(\tilde{\theta}_{6}\left(\vec{r}+\vec{r}_{0}, t\right)-\tilde{\theta}_{6}\left(\vec{r}_{0}, t\right)\right)$ and thus only probes the spatial correlations in the argument of the bond-orientational order parameter at different times.

\section{APPENDIX C: DETECTION OF GRAIN BOUNDARIES}

To extract the GBs and their misorientation $\theta$, the grains are first detected using a procedure similar to the one used by Dillmann et al. [29]. An example for a small part of 
(a)

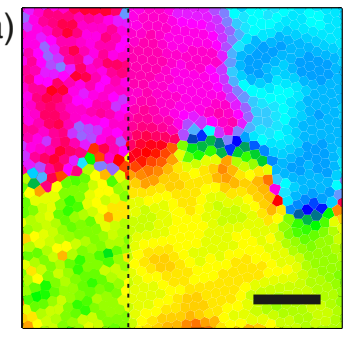

(c)

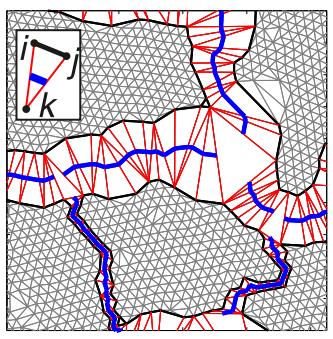

(b)

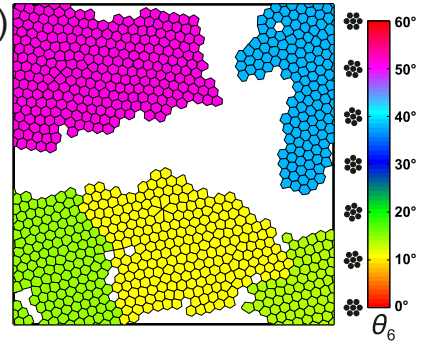

(d)

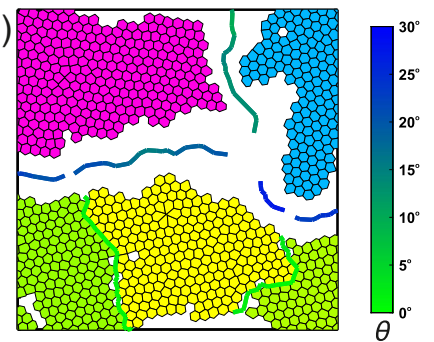

FIG. 7. (a) Local view of the smoothed orientation map $\tilde{\theta}_{6}$. The inset on the left of the snapshot shows the raw orientation map $\theta_{6}$ before smoothing. The scale bar represents $20 \mu \mathrm{m}$. (b) The output of the grain detection algorithm adapted from Dillmann et al. [29]. Each grain is colored according to the average $\tilde{\theta}_{6}$ of the particles within it. (c) Delaunay triangulation of the crystal-like particles. The grain edges are colored in black, the GB connectors in red, and the GB segments in blue. The inset shows the construction of a GB segment involving three particles $i, j$, and $k$ (see text). (d) The output of the grain detection together with the GB segments colored according to their misorientation as indicated by the color bar.

the system is shown in Fig. 7(a). The set of crystal-like particles is defined as those particles for which $\left|\tilde{\psi}_{6}\right|$ is greater that 0.7 . If a crystal-like particle has at least three crystal-like neighbors whose $\tilde{\theta}_{6}$ values differ by less than $0.75^{\circ}$, then these particles belong to the same grain. A grain containing less than 19 particles is discounted as this corresponds to the size of a cluster formed by a particle and its two shells of nearest neighbors [61]. The output is shown in Fig. 7(b), where the particles of each grain have been colored according to the average $\tilde{\theta}_{6}$ value of the particles within that grain.

Next, the GBs are determined by constructing the Delaunay triangulation of the crystal-like particles only, as shown in Fig. 7(c). From this, the triangulation edges forming the envelope of the grains are detected and form the grain edges, such as $i-j$ in the inset Fig. 7(c). The triangulation edges that connect the particles belonging to two neighboring grains are detected as well. These edges are called the "GB connectors", such as $k-i$ or $k-j$ in the inset of Fig. 7(c). Linking the centers of two consecutive GB connectors such as $k-i$ and $k-j$ gives a "GB segment," as seen in the inset of Fig. 7(c). It is clear from Fig. 7(c) that the GB segments determined by such a method provide a reasonably good representation of the GBs in the system.

To compute the misorientation of GBs, one starts by defining the misorientation of a GB connector as

$\theta_{i k}=\min \left(\left|\tilde{\theta}_{6, i}-\tilde{\theta}_{6, k}\right|, 60^{\circ}-\left|\tilde{\theta}_{6, i}-\tilde{\theta}_{6, k}\right|\right) \in\left[0,30^{\circ}\right]$,

where $\tilde{\theta}_{6, i}$ is the smoothed orientation at the site $i$. Note that the expression of $\theta_{i k}$ respects the symmetry $\theta \rightarrow 60^{\circ}-\theta$ of hexagonal closed-packed crystals. The misorientation $\theta_{k i j}$ associated to a GB segment involving the two GB connectors $k-i$ and $k-j$ is then calculated via

$$
\theta_{k i j}=\min \left(\left|\frac{\theta_{k i}+\theta_{k j}}{2}\right|, 60^{\circ}-\left|\frac{\theta_{k i}+\theta_{k j}}{2}\right|\right),
$$

which lies in the range $0^{\circ}-30^{\circ}$ as well. The GB segments, colored according to their misorientation, are plotted in Fig. 7(d) to directly visualize the local misorientation of the GBs. One can see that the algorithm is able to detect most GBs present in the snapshot, including two GBs with a small misorientation $\simeq 5^{\circ}$. The reason why LAGBs appear wider than SAGBs in Figs. 7(b) and 7(d) comes from the fact that LAGBs have a higher dislocation density than SAGBs $[9,25,62]$. As a consequence, crystalline order is more disrupted by a LAGB, which leads to lower values of the modulus of $\psi_{6}$ and a noisier orientation field, and thus results in more GB particles being detected in a LAGB than in a SAGB.

The GB inclination is obtained using the GB construction procedure shown in Figs. 8(a)-8(c) [25,62]. Starting with a perfect lattice of primitive vectors $\vec{a}_{1}$ and $\vec{a}_{2}$ [63], one draws a line that corresponds to the GB, thus separating two grains labeled (1) and (2), as illustrated in Fig. 8(a). The inclination $\psi$ is then defined as the angle between $\vec{a}_{1}$ and the GB line [see Fig. 8(a)]. The GB is then obtained by rotating grains (1) and (2) by $-\theta / 2$ and $+\theta / 2$, respectively, where $\theta$ is the misorientation [see Fig. 8(b)]. The situation actually encountered in an experiment is that illustrated in Fig. 8(c), where grain (1) has an orientation $\alpha$ with respect to the $x$ axis. In this case, the inclination can be measured using

$$
\psi=\beta-\theta / 2-\alpha,
$$

where $\beta$ is the direction of the GB line with respect to the $x$ axis [see Fig. 8(c)]. For a GB segment as considered in Fig. 7(c), the inclination is thus

$$
\psi_{k i j}=\beta_{k i j}-\theta_{k i j} / 2-\alpha_{k i j},
$$

where $\theta_{k i j}$ is the misorientation as obtained from Eq. (C2); $\alpha_{k i j}=\min \left(\tilde{\theta}_{6, k}, \tilde{\theta}_{6, i}, \tilde{\theta}_{6, j}\right)$; and $\beta_{k i j}$ is the direction of the GB segment. However, the outcome of $\beta_{k i j}$ would be subject to noise from the thermal fluctuations of the detected GB. Instead, the local direction at a point of the GB is computed from the positions of GB points located 
(a)

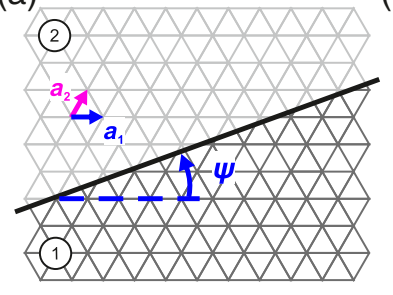

(b)
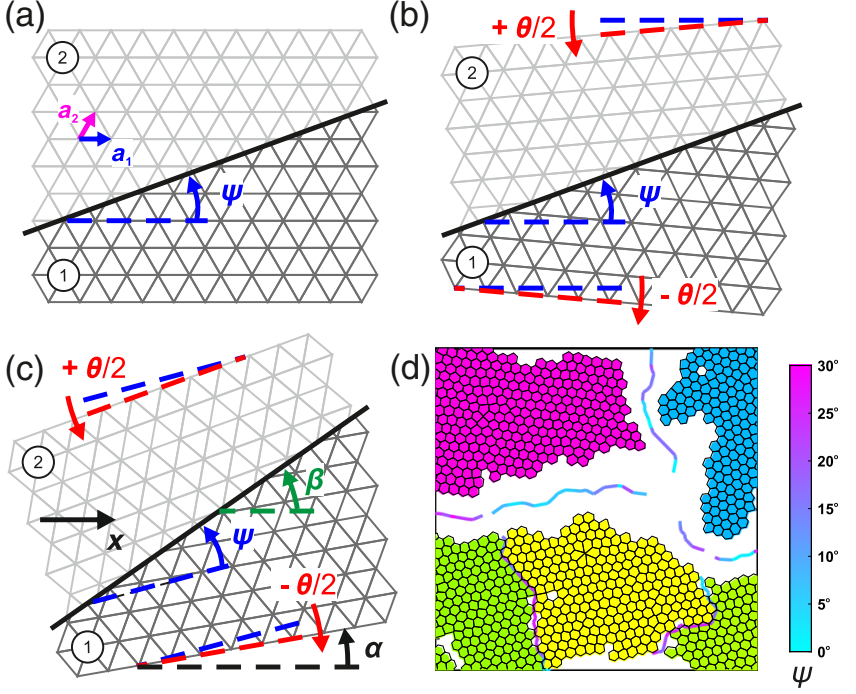

FIG. 8. (a) Definition of the GB inclination $\psi$ from the GB line (black line) in the starting lattice. (b) Construction of the GB by the rotation of grains (1) and (2) by $\mp \theta / 2$, where $\theta$ is the misorientation. (c) Schematic view of a GB as encountered in an experiment, where $\alpha$ is the orientation of grain (1) and $\beta$ the tangent direction of the GB with respect to the reference $x$-axis. (d) Similar view as in Fig. 7(d) with the GB segments colored according to their inclination as indicated by the color bar.

within some cutoff distance from the point of interest, using principal component analysis [39]. Here, we choose the cutoff distance to be $2 \sigma$, which matches our cutoffs used for the $\psi_{6}$ smoothing and the grain detection. As by symmetry, $\psi_{k i j}$ lies within the range $0^{\circ}-30^{\circ}$, and we define a reduced inclination

$\bar{\psi}_{k i j}=\min \left[\psi_{k i j} \bmod 60^{\circ}, 60^{\circ}-\left(\psi_{k i j} \bmod 60^{\circ}\right)\right]$,

which is referred to as "the inclination" in this work. The inclination of the GB segment as determined by this procedure can be directly visualized in Fig. 8(d).

Finally, the GBCD is determined from the length $l$ of each GB segment. The lengths of the GB segments with a misorientation in the interval $[\theta ; \theta+\delta \theta]$ and an inclination in the interval $[\psi ; \psi+\delta \psi]$ are added together and this sum is denoted $l(\theta, \psi, t)$. Dividing $l(\theta, \psi, t)$ by the total length of GB segments yields the value of the GBCD, $p(\theta, \psi, t)$, according to Eq. (5). The values of $\delta \theta$ and $\delta \psi$ are set to $0.75^{\circ}$, consistently with the grain detection step.

[1] A. J. Bray, Theory of Phase-Ordering Kinetics, Adv. Phys. 43, 357 (1994).

[2] L. F. Cugliandolo, Topics in Coarsening Phenomena, Physica (Amsterdam) 389A, 4360 (2010).

[3] P. M. Chaikin and T. C. Lubensky, Principles of Condensed Matter Physics (Cambridge Univ. Press, Cambridge, England, 2000).
[4] D. R. Nelson, Defects and Geometry in Condensed Matter Physics (Cambridge University Press, Cambridge, England, 2002).

[5] C. Harrison, D. H. Adamson, Z. Cheng, J. M. Sebastian, S. Sethuraman, D. A. Huse, R. A. Register, and P. M. Chaikin, Mechanisms of Ordering in Striped Patterns, Science 290, 1558 (2000).

[6] E. A. G. Jamie, R. P. A. Dullens, and D. G. A. L. Aarts, Spinodal Decomposition of a Confined Colloid-Polymer System, J. Chem. Phys. 137, 204902 (2012).

[7] H. Orihara and Y. Ishibashi, Dynamics of Disclinations in Twisted Nematics Quenched Below the Clearing Point, J. Phys. Soc. Jpn. 55, 2151 (1986).

[8] A. Sicilia, J. J. Arenzon, I. Dierking, A. J. Bray, L. F. Cugliandolo, J. Martínez-Perdiguero, I. Alonso, and I. C. Pintre, Experimental Test of Curvature-Driven Dynamics in the Phase Ordering of a Two Dimensional Liquid Crystal, Phys. Rev. Lett. 101, 197801 (2008).

[9] A. Rollett, F. J. Humphreys, G. S. Rohrer, and M. Hatherly, Recrystallization and Related Annealing Phenomena (Elsevier, New York, 2004).

[10] S. Yip, Nanocrystals: The Strongest Size, Nature (London) 391, 532 (1998).

[11] J. Schiøtz, F. D. Di Tolla, and K. W. Jacobsen, Softening of Nanocrystalline Metals at Very Small Grain Sizes, Nature (London) 391, 561 (1998).

[12] L. Lu, X. Chen, X. Huang, and K. Lu, Revealing the Maximum Strength in Nanotwinned Copper, Science 323, 607 (2009).

[13] M. P. Anderson, D. J. Srolovitz, G. S. Grest, and P. S. Sahni, Computer Simulation of Grain Growth-I. Kinetics, Acta Mater. 32, 783 (1984).

[14] D. J. Srolovitz, M. P. Anderson, P. S. Sahni, and G. S. Grest, Computer simulation of grain growth-II. Grain Size Distribution Topology, and Local Dynamics, Acta Mater. 32, 793 (1984).

[15] A. J. Haslam, S. R. Phillpot, D. Wolf, D. Moldovan, and H. Gleiter, Mechanisms of Grain Growth in Nanocrystalline FCC Metals by Molecular-Dynamics Simulation, Mater. Sci. Eng. A 318, 293 (2001).

[16] A. Adland, Y. Xu, and A. Karma, Unified Theoretical Framework for Polycrystalline Pattern Evolution, Phys. Rev. Lett. 110, 265504 (2013).

[17] D. S. Aidhy, Y. Zhang, and W. J. Weber, A Fast GrainGrowth Mechanism Revealed in Nanocrystalline Ceramic Oxides, Scr. Mater. 83, 9 (2014).

[18] S. Schmidt, S. F. Nielsen, C. Gundlach, L. Margulies, X. Huang, and D. J. Jensen, Watching the Growth of Bulk Grains During Recrystallization of Deformed Metals, Science 305, 229 (2004).

[19] S. Schmidt, U. L. Olsen, H. F. Poulsen, H. O. Sørensen, E. M. Lauridsen, L. Margulies, C. Maurice, and D. J. Jensen, Direct Observation of 3-D Grain Growth in Al-0.1\% Mn, Scr. Mater. 59, 491 (2008).

[20] D. Moldovan, V. Yamakov, D. Wolf, and S. R. Phillpot, Scaling Behavior of Grain-Rotation-Induced Grain Growth, Phys. Rev. Lett. 89, 206101 (2002).

[21] J. W. Cahn and J. E. Taylor, A Unified Approach to Motion of Grain Boundaries, Relative Tangential Translation along 
Grain Boundaries, and Grain Rotation, Acta Mater. 52, 4887 (2004).

[22] E. A. Holm and S. M. Foiles, How Grain Growth Stops: A Mechanism for Grain-Growth Stagnation in Pure Materials, Science 328, 1138 (2010).

[23] Z. T. Trautt and Y. Mishin, Grain Boundary Migration and Grain Rotation Studied by Molecular Dynamics, Acta Mater. 60, 2407 (2012).

[24] S. M. Allen and J. W. Cahn, A Microscopic Theory for Antiphase Boundary Motion and Its Application to Antiphase Domain Coarsening, Acta Mater. 27, 1085 (1979).

[25] G. Gottstein and L.S. Shvindlerman, Grain Boundary Migration in Metals: Thermodynamics, Kinetics, Applications (CRC Press, Boca Raton, Florida, 1999).

[26] P. A. Deymier, J. O. Vasseur, and L. Dobrzynski, Anomalous Exponent in the Kinetics of Grain Growth with Anisotropic Interfacial Energy, Phys. Rev. B 55, 205 (1997).

[27] G. T. Higgins, Grain-Boundary Migration and Grain Growth, Metal science 8, 143 (1974).

[28] K. Yoshizawa, T. Okuzono, T. Koga, T. Taniji, and J. Yamanaka, Exclusion of Impurity Particles During Grain Growth in Charged Colloidal Crystals, Langmuir 27, 13420 (2011).

[29] P. Dillmann, G. Maret, and P. Keim, Polycrystalline Solidification in a Quenched 2D Colloidal System, J. Phys. Condens. Matter 20, 404216 (2008).

[30] C. Harrison, D. E. Angelescu, M. Trawick, Z. Cheng, D. A. Huse, P. M. Chaikin, D. A. Vega, J. M. Sebastian, R. A. Register, and D. H. Adamson, Pattern Coarsening in a 2D Hexagonal System, Europhys. Lett. 67, 800 (2004).

[31] D. A. Vega, C. K. Harrison, D. E. Angelescu, M. L. Trawick, D. A. Huse, P. M. Chaikin, and R. A. Register, Ordering Mechanisms in Two-Dimensional Sphere-Forming Block Copolymers, Phys. Rev. E 71, 061803 (2005).

[32] L. R. Gómez, E. M. Vallés, and D. A. Vega, Lifshitz-Safran Coarsening Dynamics in a 2D Hexagonal System, Phys. Rev. Lett. 97, 188302 (2006).

[33] J. C. Crocker and D. G. Grier, Methods of Digital Video Microscopy for Colloidal Studies, J. Colloid Interface Sci. 179, 298 (1996).

[34] S. Gokhale, K. H. Nagamanasa, R. Ganapathy, and A. K. Sood, Grain Growth and Grain Boundary Dynamics in Colloidal Polycrystals, Soft Matter 9, 6634 (2013).

[35] S. Deutschländer, P. Dillmann, G. Maret, and P. Keim, Kibble-Zurek Mechanism in Colloidal Monolayers, Proc. Natl. Acad. Sci. U.S.A. 112, 6925 (2015).

[36] E. P. Bernard and W. Krauth, Two-Step Melting in Two Dimensions: First-Order Liquid-Hexatic Transition, Phys. Rev. Lett. 107, 155704 (2011).

[37] M. Engel, J. A. Anderson, S. C. Glotzer, M. Isobe, E. P. Bernard, and W. Krauth, Hard-Disk Equation of State: First-Order Liquid-Hexatic Transition in Two Dimensions with Three Simulation Methods, Phys. Rev. E 87, 042134 (2013).

[38] A. L. Thorneywork, J. L. Abbott, D. G. A. L. Aarts, and R.P. A. Dullens, Two-Dimensional Melting of Colloidal Hard Spheres, Phys. Rev. Lett. 118, 158001 (2017).
[39] F. A. Lavergne, D. G. A. L. Aarts, and R. P. A. Dullens, Determining Local Geometrical Features of Grain Boundaries from Microscopy, J. Phys. Condens. Matter 27, 194117 (2015).

[40] F. A. Lavergne, S. Diana, D. G. A. L. Aarts, and R. P. A. Dullens, Equilibrium Grain Boundary Segregation and Clustering of Impurities in Colloidal Polycrystalline Monolayers, Langmuir 32, 12716 (2016).

[41] O. V. Yazyev and Y. P. Chen, Polycrystalline Graphene and Other Two-Dimensional Materials, Nat. Nanotechnol. 9, 755 (2014).

[42] K. Barmak, E. Eggeling, M. Emelianenko, Y. Epshteyn, D. Kinderlehrer, R. Sharp, and S. Ta'asan, Critical Events, Entropy, and the Grain Boundary Character Distribution, Phys. Rev. B 83, 134117 (2011).

[43] E. A. Holm, G. S. Rohrer, S. M. Foiles, A. D. Rollett, H. M. Miller, and D. L. Olmsted, Validating Computed Grain Boundary Energies in FCC Metals Using the Grain Boundary Character Distribution, Acta Mater. 59, 5250 (2011).

[44] C. Herring, in The Physics of Powder Metallurgy, edited by W. E. Kingston (McGraw-Hill, New York, 1951).

[45] S. M. Foiles and J. J. Hoyt, Computation of Grain Boundary Stiffness and Mobility from Boundary Fluctuations, Acta Mater. 54, 3351 (2006).

[46] E. A. Holm, G. N. Hassold, and M. A. Miodownik, On Misorientation Distribution Evolution during Anisotropic Grain Growth, Acta Mater. 49, 2981 (2001).

[47] A. L. Thorneywork, R. Roth, D. G. A. L. Aarts, and R. P. A. Dullens, Radial Distribution Functions in a Two-dimensional Binary Colloidal Hard Sphere System, J. Chem. Phys. 140, 161106 (2014).

[48] M. P. O. Loureiro, J. J. Arenzon, L. F. Cugliandolo, and A. Sicilia, Curvature-Driven Coarsening in the TwoDimensional Potts Model, Phys. Rev. E 81, 021129 (2010).

[49] T. Ohta, D. Jasnow, and K. Kawasaki, Universal Scaling in the Motion of Random Interfaces, Phys. Rev. Lett. 49, 1223 (1982).

[50] H. Toyoki, Structure Factors of Vector-Order-Parameter Systems Containing Random Topological Defects, Phys. Rev. B 45, 1965 (1992).

[51] R. E. Blundell and A. J. Bray, Phase-Ordering Dynamics of the $O(n)$ Model: Exact Predictions and Numerical Results, Phys. Rev. E 49, 4925 (1994).

[52] K. Zahn, R. Lenke, and G. Maret, Two-Stage Melting of Paramagnetic Colloidal Crystals in Two Dimensions, Phys. Rev. Lett. 82, 2721 (1999).

[53] Y. Peng, Z. Wang, A. M. Alsayed, A. G. Yodh, and Y. Han, Melting of Colloidal Crystal Films, Phys. Rev. Lett. 104, 205703 (2010).

[54] T. O. E. Skinner, D. G. A. L. Aarts, and R. P. A. Dullens, Grain-Boundary Fluctuations in Two-Dimensional Colloidal Crystals, Phys. Rev. Lett. 105, 168301 (2010).

[55] P. Y. Huang, S. Kurasch, J. S. Alden, A. Shekhawat, A. A. Alemi, P. L. McEuen, J. P. Sethna, U. Kaiser, and D. A. Muller, Imaging Atomic Rearrangements in TwoDimensional Silica Glass: Watching Silica's Dance, Science 342, 224 (2013). 
[56] S. Wachter, D. K. Polyushkin, O. Bethge, and T. Mueller, A Microprocessor Based on a Two-Dimensional Semiconductor, Nat. Commun. 8, 14948 (2017).

[57] S. Najmaei, Z. Liu, W. Zhou, X. Zou, G. Shi, S. Lei, B. I. Yakobson, J.-C. Idrobo, P. M. Ajayan, and J. Lou, Vapour Phase Growth and Grain Boundary Structure of Molybdenum Disulphide Atomic Layers, Nat. Mater. 12, 754 (2013).

[58] Y. Lin and J. W. Connell, Advances in 2D Boron Nitride Nanostructures: Nanosheets, Nanoribbons, Nanomeshes, and Hybrids with Graphene, Nanoscale 4, 6908 (2012).

[59] A. L. Thorneywork, R. E. Rozas, R. P. A. Dullens, and J. Horbach, Effect of Hydrodynamic Interactions on
Self-Diffusion of Quasi-Two-Dimensional Colloidal Hard Spheres, Phys. Rev. Lett. 115, 268301 (2015).

[60] D. Blair and E. Dufresne, The Matlab Particle Tracking Code Repository, retrieved from http://physics.georgetown .edu/matlab/.

[61] This criterion actually matches the cutoff for the smoothing of the $\psi_{6}$ field as can be seen from Fig. 6(b).

[62] W. T. Read and W. Shockley, Dislocation Models of Crystal Grain Boundaries, Phys. Rev. 78, 275 (1950).

[63] C. Kittel, Introduction to Solid State Physics (Wiley, New York, 2005). 